\title{
Motional Dynamical Decoupling for Interferometry with Macroscopic Particles
}

\author{
Julen S. Pedernales $\odot,{ }^{1}$ Gavin W. Morley $\odot,{ }^{2}$ and Martin B. Plenio ${ }^{1}$ \\ ${ }^{1}$ Institut für Theoretische Physik und IQST, Albert-Einstein-Allee 11, Universität Ulm, D-89081 Ulm, Germany \\ ${ }^{2}$ Department of Physics, University of Warwick, Gibbet Hill Road, Coventry CV4 7AL, United Kingdom
}

(Received 10 June 2019; accepted 29 May 2020; published 9 July 2020)

\begin{abstract}
We extend the concept of dynamical decoupling from spin to mechanical degrees of freedom of macroscopic objects, for application in interferometry. In this manner, the superposition of matter waves can be made resilient to many important sources of noise when these are driven along suitable paths in space. As a concrete implementation, we present the case of levitated (or free falling) nanodiamonds hosting a color center in a magnetic field gradient. We point out that these interferometers are inherently affected by diamagnetic forces, which restrict the separation of the superposed states to distances that scale with the inverse of the magnetic field gradient. Periodic forcing of the mechanical degree of freedom is shown to overcome this limitation, achieving a linear-in-time growth of the separation distance independent of the magnetic field gradient, while simultaneously protecting the coherence of the superposition from environmental perturbations.
\end{abstract}

DOI: 10.1103/PhysRevLett.125.023602

Introduction.-Matter-wave interferometry provides a remarkably sensitive tool for probing minute forces and, potentially, the foundations of quantum physics by making use of interference between spatially separated matter waves. The sensitivity of such interferometers might be improved by increasing the size of the object in superposition, however, this will typically entail a concomitant increase in its sensitivity to uncontrolled sources of noise. A key question now arises naturally: how can one mitigate the impact of noise in macroscopic matter-wave interferometers, while maintaining sensitivity to a desired signal? This challenge is reminiscent of that of quantum sensing using the electron spin of nitrogen vacancy (NV) centers, where a wide variety of dynamical decoupling schemes have been developed that filter out slow noise, while retaining sensitivity to a signal at a particular frequency [1-6]. Finding analogous dynamical decoupling protocols that address spatial degrees of freedom could provide significant sensitivity improvements in interferometry experiments with massive particles, as well as open the door to tests of the linearity of quantum mechanics at larger scales [7].

Noise canceling techniques in atomic matter-wave interferometry have been explored already, especially regarding the suppression of static noise sources in atom interferometers [8-10]. These techniques rely on path symmetrization schemes that double the flight time of the atoms and

Published by the American Physical Society under the terms of the Creative Commons Attribution 4.0 International license. Further distribution of this work must maintain attribution to the author(s) and the published article's title, journal citation, and DOI. invert the interference paths in the second half of the protocol. Hence, they require that the noise is static during the process of splitting and recombination of the wave function, which in turn limits the achievable separation distance. In the light of this, it would be desirable to find a dynamical decoupling technique that is incorporated into the wave function splitting mechanism, such that the splitting of the wave function can be continued beyond the fluctuation times of the noise until the desired separation has been reached.

Mesoscopic matter-wave interferometers [11,12] based on Bose-Einstein condensates [13], fullerenes [14], or more recently, macromolecules containing up to $25000 \mathrm{Da}$ [1517] have been implemented that can bring objects with an increasing number of atoms to coherent superposition. In parallel, a top-down approach is also being pursued aiming at achieving a leap in the size of the interfered object, with a variety of optomechanical systems proposed as potential macroscopic matter-wave interferometers [18-27]. Regarding this second approach, we are interested, in particular, in setups where the interaction of a strong magnetic field gradient with a NV center is used to split the spatial wave function of the host nanodiamond, which can either be levitated or in free fall. This represents a promising platform, on the one hand, due to the remarkable coherence properties of $\mathrm{NV}$ centers even at room temperature $[28,29]$ - in part, due to a mature understanding of NMR techniques applied to NV centers for the purposes of sensing and metrology [30,31] —and, on the other hand, due to the recent and rapid development of the field that has demonstrated an increasing degree of controllability of NV centers in nanodiamonds that are levitated either by optical means [32-35], with ion traps [36-40], or in magnetogravitational 
traps [41,42]. However, these setups suffer from the impact of diamagnetic forces, which are intrinsic to the use of magnetic field gradients and diamond, and which, if not acted upon, limit the reachable separation distance of the superposition.

In this Letter, we introduce a novel pulse sequence that affects motional dynamics of matter-wave interferometers based on nanodiamonds hosting a NV center. Akin to pulsed dynamical decoupling, our protocol suppresses those system-environment interactions that fluctuate slower than the interpulse spacing. The sequence is integrated into the interferometric protocol, actively protecting the spatial degrees of freedom of the nanoparticle during the process of splitting and, at the same time, enhancing the reachable separation distance via a resonant mechanism that addresses the impact of diamagnetic forces. As an added benefit, when applied resonantly with the trapping frequency, this results in a linear-in-time growth of the separation distance, exceeding the maximum separation otherwise imposed by the diamagnetism of diamond.

Setup and protocol. - We consider an irregularly shaped nanodiamond containing a single negatively charged NV center that is localized at some random distance from the center of mass. The diamond is initially levitated and cooled down to an internal temperature on the order of $1 \mathrm{~K}$. In the $x$ direction, a magnetic field gradient is applied such that the magnetic field is aligned with the axis of the NV center, exerting a spin-dependent force on the NV; see Fig. 1(a). The motion in all the three Cartesian coordinates is uncoupled, and thus, the analysis of the dynamics in the $x$ direction is valid for both the case in which the diamond remains trapped and that in which the trapping fields are relaxed and the diamond falls freely along the $z$ direction due to the action of gravity. For simplicity, we consider here the second case. In the absence of perturbing forces, the dynamics in the $x$ direction is governed by the Hamiltonian

$$
\begin{aligned}
H_{x} & =\frac{1}{2 M} \hat{P}_{x}^{2}-\frac{\chi_{V} V B^{\prime 2}}{2 \mu_{0}} \hat{x}^{2}+\hbar \gamma_{e} B^{\prime} \hat{S}_{z} \hat{x}+\hbar D \hat{S}_{z}^{2} \\
& =\hbar \omega \hat{a}^{\dagger} \hat{a}+\hbar \lambda\left(a+a^{\dagger}\right) \hat{S}_{z}+\hbar D \hat{S}_{z}^{2},
\end{aligned}
$$

where the second term in the first line of Eq. (1) represents the diamagnetic energy of the diamond in the presence of a constant magnetic field gradient $\mathbf{B}=B^{\prime} x \mathbf{e}_{z}^{(\mathrm{NV})}$, with $B^{\prime}$ as the magnitude of the gradient, and $\mathbf{e}_{z}^{(\mathrm{NV})}$ as a unit vector aligned with the direction of the NV axis. Here, $\chi_{V}=$ $-2.2 \times 10^{-5}$ and $V$ are, respectively, the volume magnetic susceptibility and the volume of the diamond, and $\mu_{0}$ is the vacuum permeability. For materials with a negative magnetic susceptibility like diamond, this results in an effective harmonic trapping with oscillation frequency $\omega=$ $\sqrt{\left(-\chi_{V} / \rho_{D} \mu_{0}\right)} B^{\prime}$, where $\rho_{D}=3510 \mathrm{Kg} / \mathrm{m}^{3}$ is the mass density of diamond, which has been used recently to demonstrate the trapping and cooling of nanodiamonds with magnetic traps $[41,42]$. The third term in the first line (a)

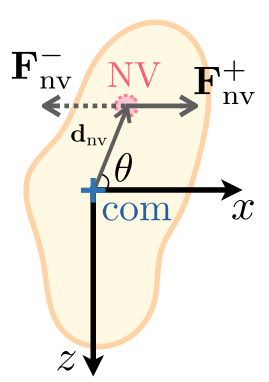

(b)

(c)

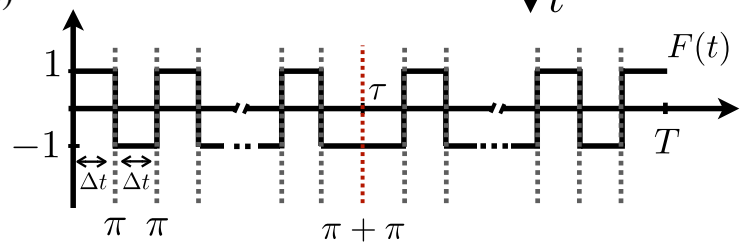

FIG. 1. Schematics of setup and dynamics. (a) An irregularly shaped diamond is depicted in pale yellow, with a NV (pink) sitting at a position $d_{\mathrm{NV}}$ from the center of mass (blue). The forces acting on the NV due to the magnetic field gradient are also indicated. (b) The oscillations amplified by resonant modulation associated with spin-up (down) are shown in red (blue). The amplitude of the oscillations grows linearly in time as $D_{\max }(t)$, depicted in the figure with a diagonal gray line. Vertical lines indicate the equilibrium positions of the two oscillators. The oscillations corresponding to the spin-up state in the absence of the pulse sequence are shown in green. (c) The function $F(t)$ with vertical dashed lines indicating $\pi$ pulses. An additional $\pi$ pulse (red dashed line) is applied at time $\tau$, when the desired separation has been reached, in order to reverse the dynamics and bring the diamond back to its initial state at time $T=2 \tau$. Therefore, at time $\tau$, two $\pi$ pulses are applied or, equivalently, none.

of Eq. (1) represents the energy of the NV spin in the magnetic field gradient, which results in a spin-motion coupling of strength $\lambda=\gamma_{e} \sqrt{(\hbar / 2 M \omega)} B^{\prime}$, where $\gamma_{e}=$ $(2 \pi) 28 \mathrm{GHz} / \mathrm{T}$ is the electronic gyromagnetic ratio and $M$ is the mass of the diamond. The last term in both lines of Eq. (1) represents the zero-field splitting of the NV center, with $D=(2 \pi) 2.8 \mathrm{GHz}$.

The Hilbert space of the ground-state electron spin of the $\mathrm{NV}$ can be spanned in terms of the eigenstates of the $\hat{S}_{z}$ operator, $\left\{\left|m_{s}\right\rangle\right\}=\{|+\rangle,|-\rangle,|0\rangle\}$, and the Hamiltonian reformulated in terms of displaced ladder operators associated with each of the NV spin states

$$
\begin{aligned}
H_{x}= & \hbar \omega a_{+}^{\dagger} a_{+}|+\rangle\left\langle+\left|+\hbar \omega a_{-}^{\dagger} a_{-}\right|-\right\rangle\langle-| \\
& +\hbar \omega\left(a^{\dagger} a+\lambda^{2} / \omega^{2}-D / \omega\right)|0\rangle\langle 0| .
\end{aligned}
$$

Here, $a_{ \pm}=a \pm \lambda / \omega$ and we have shifted the total energy by a constant $\hbar \lambda^{2} / \omega-D$, with no loss of generality. The system is, therefore, a set of three harmonic oscillators each associated with a state of the NV spin and that oscillate, 
with the same frequency, around three different equilibrium positions, namely, $x_{\mathrm{eq}}^{0}=0$ and $x_{\mathrm{eq}}^{ \pm}= \pm 2 x_{0} \lambda / \omega$, with $x_{0}=\sqrt{\hbar /(2 M \omega)}$.

Our goal is to put the center of mass of the diamond in a coherent superposition of two states that are spatially separated by the largest possible distance. Let us assume that the nanodiamond is initially in an unspecified motional state $\rho_{m}$ and that we place the NV in a superposition of $| \pm\rangle$ states. The system is in an even superposition of two components, each of which oscillates around spatially separated equilibrium positions. An operator can be defined in the Heisenberg picture whose expectation value corresponds to the separation distance between these two oscillators, namely,

$$
\delta \hat{x}(t)=U_{+}^{\dagger} \hat{x} U_{+}-U_{-}^{\dagger} \hat{x} U_{-}=\Delta x_{\mathrm{eq}}(\cos \omega t-1),
$$

where $U_{ \pm}=e^{-i \omega a_{ \pm}^{\dagger} a_{ \pm} t}$, and $\Delta x_{\mathrm{eq}}=4 x_{0} \lambda / \omega=2 \hbar \gamma_{e} \mu_{0} /$ $\left(-\chi_{V} V B^{\prime}\right)$ is the separation distance between the equilibrium positions of the two oscillators. The system will periodically split and reunite such that the maximal separation distance

$$
D_{\max }=2 \Delta x_{\mathrm{eq}}=\frac{4 \hbar \gamma_{e} \mu_{0}}{-\chi_{V}} \frac{1}{V B^{\prime}}
$$

is reached for the first time at half an oscillation period and does not grow in time, contrary to predictions of previous proposals $[43,44]$.

Indeed, contrary to previous analyses where diamagnetic forces were ignored and the separation distance was predicted to increase with the magnetic field gradient $[43,44]$, we find that the separation distance decreases with the magnetic field gradient. Notice, however, that, for proposals with levitated nanodiamonds where a trapping mechanism stronger than the diamagnetic confinement is employed, the reachable separation distance will still grow with the magnetic field gradient. This is the case for optically trapped particles, where neglecting the diamagnetic forces is justified $[45,46]$, albeit, for the same value of the magnetic field gradient, the reachable separation distances will be considerably smaller than in the case of pure diamagnetic trapping. Remarkably, in our case, the maximum separation distance is independent of the initial motional state, i.e., initial displacement from $x=0$ and velocity, and is determined only by the distance between the equilibrium positions. This indicates that ground-state cooling of the motional degrees of freedom is not required for such a scheme.

Motional dynamical decoupling.-We consider the same setup but in the presence of a sequence of $\pi$ pulses that are applied between states $|+\rangle$ and $|-\rangle$ of the NV center during the fall of the diamond. In the presence of these pulses, Hamiltonian (1) is modified as
$H_{x}=\hbar \omega a^{\dagger} a+\hbar \lambda\left(a^{\dagger}+a\right) S_{z}+\hbar \Omega(t) \sigma_{x}+\hbar \eta(t)\left(a+a^{\dagger}\right)$,

where $\Omega(t)$ is a time-dependent Rabi frequency, which has value 0 in between the pulses and some fixed value $\Omega=\pi /(2 \tau)$ while a pulse of duration $\tau$ is being applied, and $\sigma_{x}=|+\rangle\langle-|+$ H.c. The last term in Eq. (5) represents the presence of an uncontrolled potential energy term that is linear in the dimension of the superposition and that may fluctuate in time, corresponding to noise in the form of a fluctuating force in direction $x$.

Moving into an interaction picture with respect to $\hbar \omega a^{\dagger} a+\hbar \Omega(t) \sigma_{x}$ and assuming that the pulses are instantaneous compared to any other frequencies in the system, we obtain

$$
H_{x}^{\text {int }}=\hbar\left[F(t) \lambda S_{z}+\eta(t)\right]\left(a e^{-i \omega t}+a^{\dagger} e^{i \omega t}\right),
$$

where the effect of the $\pi$ pulses is to flip the sign of operator $S_{z}$. This is captured by the function $F(t)$, which is a piecewise constant function taking only values 1 or -1 ; see Fig. 1(a).

The unitary-evolution operator associated with the timedependent Hamiltonian (6) can be determined in the Magnus expansion, where terms of order 3 and higher vanish identically. Thus, $U(t)=e^{\Omega^{(1)}+\Omega^{(2)}}$, with

$$
\begin{gathered}
\Omega^{(1)}=\left(\alpha_{t} a-\alpha_{t}^{*} a^{\dagger}\right) S_{z}+\beta_{t} a-\beta_{t}^{*} a^{\dagger}, \\
\Omega^{(2)}=\gamma_{t} S_{z}+\delta_{t},
\end{gathered}
$$

and

$$
\begin{gathered}
\alpha_{t}=-i \lambda \int_{0}^{t} d t_{1} F\left(t_{1}\right) e^{-i \omega t_{1}} \\
\gamma_{t}=-i \lambda \int_{0}^{t} d t_{1} F\left(t_{1}\right)\left(\int_{0}^{t_{1}} d t_{2} \eta\left(t_{2}\right) \sin \left[\omega\left(t_{1}-t_{2}\right)\right]\right. \\
\left.-\int_{t_{1}}^{t} d t_{2} \eta\left(t_{2}\right) \sin \left[\omega\left(t_{1}-t_{2}\right)\right]\right) .
\end{gathered}
$$

Terms linear in $\beta_{t}$ and $\delta_{t}$ correspond to a displacement in phase space and a global phase, respectively, and do not affect the superposition. However, terms linear in $\alpha_{t}$ and $\gamma_{t}$ result, respectively, in a spin-dependent displacement and a randomly fluctuating relative phase. Therefore, our goal will be to maximize $\alpha_{t}$ and suppress the effect of $\gamma_{t}$, in order to generate a spin-dependent displacement that is as large as possible, while attenuating the fluctuations in the relative phase of the superposition that would otherwise lead to decoherence.

The integral in $\alpha_{t}$ can be solved in the regions where $F(t)$ is constant and the results added. For a regular sequence consisting of $N$ pulses with an interpulse spacing of $\Delta t$, this 
yields at time $t_{N}=(N+1) \Delta t$ a displacement in phase space of amplitude

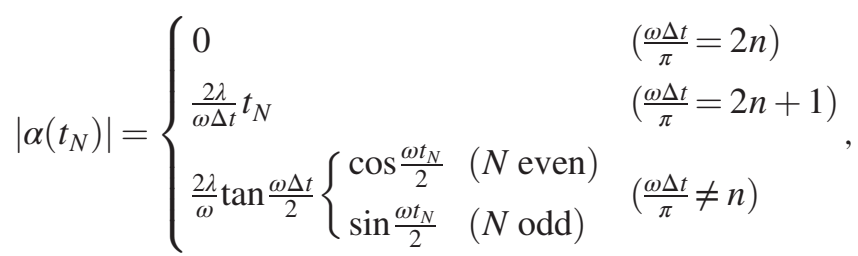

where $n$ is a natural number.

The reachable separation between the two components of the superposition is bounded for all pulse sequences except for that in which $\omega \Delta t=(2 n+1) \pi$, in which case it grows linearly in time. Moreover, in that case $\alpha$ is real and the displacement occurs in the position quadrature, resulting in a spatial separation that grows as

$$
D_{\max } / t=\frac{8}{\pi} x_{0} \lambda t=\frac{4 \hbar \gamma_{e}}{\pi V} \sqrt{\frac{\mu_{0}}{-\chi_{V} \rho_{D}}} .
$$

Remarkably, when this resonance condition is met, $D_{\max }$ does not depend on the magnetic field gradient, and the achievable separation distance is determined only by the volume of the particle and the elapsed time.

Regarding $\gamma_{t}$, we consider first the case of a static noise source $\eta(t)=\eta$ : in this case, the integral in $\gamma_{t}$ can be exactly solved

$\gamma_{t}=\frac{i \lambda \eta}{\omega} \int_{0}^{t} d t_{1} F\left(t_{1}\right)\left\{2-\cos \left(\omega t_{1}\right)-\cos \left[\omega\left(t_{1}-t\right)\right]\right\}$.

It can be made to vanish provided that the pulse sequence is antisymmetric with respect to its center $F(t-s)=-F(s)$ and that its integral is canceled $\int_{0}^{t} d t_{1} F\left(t_{1}\right)=0$, or by the concatenation of several such sequences. For the case of an evenly spaced pulse sequence, such as the one considered earlier, this is achieved by employing an odd number of pulses. Such a sequence would be an analog of pulsed dynamical decoupling sequences employed to suppress magnetic noise on spin degrees of freedom, albeit here acting as a mitigator of noise that affects the mechanical degrees of freedom of a massive particle. In the absence of a pulse sequence, the relative phase will grow as $\gamma_{t} / t=i 2 \lambda \eta / \omega$. There can be multiple physical origins for the noise term $\eta$, which will not, in general, be negligible. As an illustration, if we consider a nanodiamond of $230 \mathrm{~nm}$ radius and a magnetic field gradient of $10^{4} \mathrm{~T} / \mathrm{m}$, we find that a misalignment of the setup $\delta \theta$ will result in a gravitational relative phase $\gamma_{t} / t \approx \delta \theta 10^{11} \mathrm{~Hz}$. In the same setup, the presence of a single electron at a distance of $500 \mu \mathrm{m}$, for example, at the surface of the vacuum chamber, which interacts with the electric dipole moment that the diamond has due to the presence of the negatively charged NV center, results in an electric relative phase $\gamma_{t} / t \approx 10 \mathrm{~Hz}$. A single electronic spin on the surface of the diamond interacting with the magnetic field gradient of the experimental setup results in $\gamma_{t} / t \approx 10^{6} \mathrm{~Hz}$. All these estimations indicate that strategies to mitigate noise like the one introduced in this Letter are essential for the observation of interference in such a setup. A more thorough analysis of these sources of noise and others, e.g., Casimir-Polder forces between the diamond and the magnets, is provided in [47].

Similar to standard pulsed dynamical decoupling, for noise that fluctuates, the suppression becomes imperfect. Nevertheless, noise will be suppressed efficiently provided that the interpulse spacing is shorter than the correlation time of the noise source $\eta(t)$. Therefore, in general, a shorter interpulse spacing is needed for the suppression of faster fluctuating noise, which in turn requires a higher magnetic field gradient in order to meet the resonance condition that maximizes $\alpha_{t}$. This would, typically, result in smaller separation distances as explained in the previous section, however, with the pulse sequence introduced here, we have made the separation distance independent of the magnetic field gradient, allowing the suppression of nonstatic noise without affecting the separation distance. It should be made clear that this independence on the magnetic gradient is the aim of our pulse sequence and not to reach larger separation distances. This is because the same separation distance can always be achieved for a given time $T$ in the absence of a sequence by setting the magnetic field gradient to the minimum value that would allow a single oscillation in time $T, B^{\prime}=\sqrt{\left(-\mu_{0} \rho_{D} / \chi_{V}\right)} 2 \pi / T$; see Eq. (4).

The proposed experiment would work as follows: The $\mathrm{NV}$ is placed in a superposition of states $| \pm\rangle$, a pulse sequence is applied that matches the resonance condition $\omega \Delta t=(2 n+1) \pi$, the wave function will split and periodically reach maximum separation distances that grow linearly in time. Once the desired separation has been reached, say, at time $\tau$, an additional $\pi$ pulse will be applied, followed by the time-reversed sequence of pulses to invert the displacement and bring the wave packets together at time $T=2 \tau$. In Fig. 1(b), a scheme of the path is shown. To ensure that the decoupling occurs, the pulse sequences in each half of the experiment should contain an odd number of pulses, the additional central pulse that reverts the dynamics results in a total $2 \pi$ pulse, or equivalently, an avoided pulse, see Fig. 1(c).

Be it in the presence of a pulse sequence or not, smaller particles achieve larger separation distances, as in both cases the separation distances are inversely proportional to the volume of the oscillator. For a particle with the volume of a $1 \mu \mathrm{m}$ radius sphere, we find a separation that grows in time as $D_{\max } / t \approx 2.3 \times 10^{-8} \mathrm{~m} / \mathrm{s}$, while a particle with a radius of $230 \mathrm{~nm}$ should result in a separation that grows as $D_{\max } / t \approx 1.8 \times 10^{-6} \mathrm{~m} / \mathrm{s}$. For a free fall time of $T=0.5 \mathrm{~s}$, we set $\tau=0.25 \mathrm{~s}$, which gives a maximal separation of 
$D_{\max } \approx 5.75 \mathrm{~nm}$ for a $1 \mu \mathrm{m}$ radius particle and a separation of $D_{\max } \approx 456 \mathrm{~nm}$ if the radius of the particle is $230 \mathrm{~nm}$. In the absence of a pulse sequence, this requires a magnetic field gradient of $B^{\prime}=181.86 \mathrm{~T} / \mathrm{m}$, while use of a pulse sequence allows us to obtain the same results independent of the magnetic field gradient, at the same time protecting the coherence of the spin and mechanical degrees of freedom of the interferometer.

Our scheme is not restricted to diamagnetically trapped particles and can be applied, in general, to any system that is described by a Hamiltonian of the form in Eq. (5), that is, harmonically trapped particles with a spin degree of freedom that couples linearly to their motion. In particular, relevant to the topic of this Letter are nanodiamonds that are levitated by means other than diamagnetic forces, such as by optical forces or in Paul traps. In these cases, the confining forces will typically be stronger and, therefore, the reachable separation distances in the absence of a pulse sequence notably smaller, $D_{\max }=4 \hbar \gamma_{e} B^{\prime} / M \omega^{2}$. Thus, such setups will strongly benefit from the design introduced here in order to go beyond this limitation and reach separation distances that grow linearly in time as $D_{\max }=\left(4 \hbar \gamma_{e} B^{\prime} / M \omega \pi\right) t$.

Conclusion.-Motional dynamical decoupling opens the door to the protection of the coherence of mechanical degrees of freedom of massive objects in matter-wave interferometers. Unlike previous approaches, the protocol introduced here is able to do this in an integrated manner with the splitting mechanisms of the interferometer, in this way not deteriorating its functionality. Not only that, for the particular case of diamond where diamagnetic forces limit the reachable separation distance between the arms of the interferometer, we provide a resonant mechanism that breaches this limitation. All in all, we believe that motional dynamical decoupling will become an essential tool in driving the sensitivity of matter-wave interferometry to the next level.

M. B. P. and J. S. P. acknowledge support by the European Research Council (ERC) via the Synergy grant BioQ (Grant No. 319130), the European Union (EU) through projects HYPERDIAMOND and AsteriQs, QuantERA via project NanoSpin, the Bundesministeriums für Bildung und Forschung (BMBF) through project DiaPol, the state of Baden-Württemberg through bwHPC, the Deutsche Forschungsgemeinschaft (DFG) through Grant No. INST 40/467-1 FUGG, and the Alexander von Humboldt Foundation through a Postdoctoral Fellowship. G. W. M. is supported by the Engineering and Physical Sciences Research Council (EPSRC Grants No. EP/T001062/1 and No. EP/M013243/1) and by the Royal Society.

[1] C. A. Ryan, J. S. Hodges, and D. G. Cory, Robust Decoupling Techniques to Extend Quantum Coherence in Diamond, Phys. Rev. Lett. 105, 200402 (2010).
[2] G. de Lange, Z. H. Wang, D. Ristè, V. V. Dobrovitski, and R. Hanson, Universal dynamical decoupling of a single solid-state spin from a spin bath, Science 330, 60 (2010).

[3] B. Naydenov, F. Dolde, L. T. Hall, C. Shin, H. Fedder, L. C. L. Hollenberg, F. Jelezko, and J. Wrachtrup, Dynamical decoupling of a single-electron spin at room temperature, Phys. Rev. B 83, 081201(R) (2011).

[4] J. M. Cai, A. Retzker, F. Jelezko, and M. B. Plenio, A largescale quantum simulator on a diamond surface at room temperature, Nat. Phys. 9, 168 (2013).

[5] C. Müller, X. Kong, J.-M. Cai, K. Melentijevic, A. Stacey, M. Markham, J. Isoya, S. Pezzagna, J. Meijer, J. Du, M. B. Plenio, B. Naydenov, L. P. McGuinness, and F. Jelezko, Nuclear magnetic resonance spectroscopy with single spin sensitivity, Nat. Commun. 5, 4703 (2014).

[6] J. Casanova, Z.-Y. Wang, J. F. Haase, and M. B. Plenio, Robust dynamical decoupling sequences for individual spin addressing, Phys. Rev. A 92, 042304 (2015).

[7] A. Bassi, K. Lochan, S. Satin, T. P. Singh, and H. Ulbricht, Models of wave-function collapse, underlying theories, and experimental tests, Rev. Mod. Phys. 85, 471 (2013).

[8] K.-P. Marzlin and J. Audretsch, State independence in atom interferometry and insensitivity to acceleration and rotation, Phys. Rev. A 53, 312 (1996).

[9] B. Dubetsky and M. A. Kasevich, Atom interferometer as a selective sensor of rotation or gravity, Phys. Rev. A 74, 023615 (2006).

[10] J. Coslovskey, G. Afek, and N. Davidson, AC atom interferometry with quantum lock-in sensing, Proc. SPIE Int. Soc. Opt. Eng. 10119, Slow Light, Fast Light, and OptoAtomic Precision Metrology X, 1011903 (2017).

[11] S. Nimmrichter and K. Hornberger, Macroscopicity of Mechanical Quantum Superposition States, Phys. Rev. Lett. 110, 160403 (2013).

[12] M. Arndt and K. Hornberger, Testing the limits of quantum mechanical superpositions, Nat. Phys. 10, 271 (2014).

[13] D. S. Hall, M. R. Matthews, C. E. Wieman, and E. A. Cornell, Measurements of Relative Phase in Two-Component Bose-Einstein Condensates, Phys. Rev. Lett. 81, 1543 (1998).

[14] M. Arndt, O. Nairz, J. Vos-Andreae, C. Keller, G. van der Zouw, and A. Zeilinger, Wave-particle duality of $\mathrm{C}_{60}$ molecules, Nature (London) 401, 680 (1999).

[15] S. Eibenberger, S. Gerlich, M. Arndt, M. Mayor, and J. Tüxen, Matter-wave interference of particles selected from a molecular library with masses exceeding 10000 amu, Phys. Chem. Chem. Phys. 15, 14696 (2013).

[16] P. Haslinger, N. Dörre, P. Geyer, J. Rodewald, S. Nimmrichter, and M. Arndt, A universal matter-wave interferometer with optical ionization gratings in the time domain, Nat. Phys. 9, 144 (2013).

[17] Y. Y. Fein, P. Geyer, P. Zwick, F. Kiałka, S. Pedalino, M. Mayor, S. Gerlich, and M. Arndt, Quantum superposition of molecules beyond 25 kDa, Nat. Phys. 15, 1242 (2019).

[18] S. Mancini, V. I. Man'ko, and P. Tombesi, Ponderomotive control of quantum macroscopic coherence, Phys. Rev. A 55, 3042 (1997).

[19] S. Bose, K. Jacobs, and P. L. Knight, Scheme to probe the decoherence of a macroscopic object, Phys. Rev. A 59, 3204 (1999). 
[20] W. Marshall, C. Simon, R. Penrose, and D. Bouwmeester, Towards Quantum Superpositions of a Mirror, Phys. Rev. Lett. 91, 130401 (2003).

[21] K. G. Libbrecht and E. D. Black, Toward Quantum-limited position measurements using optically levitated microspheres, Phys. Lett. A321, 99 (2004).

[22] T. Li, S. Kheifets, D. Medellin, and M. G. Raizen, Measurement of the instantaneous velocity of a Brownian particle, Science 328, 1673 (2010).

[23] T. Li, S. Kheifets, and M. G. Raizen, Millikelvin cooling of an optically trapped microsphere in vacuum, Nat. Phys. 7, 527 (2011).

[24] B. Pepper, R. Ghobadi, E. Jeffrey, C. Simon, and D. Bouwmeester, Optomechanical Superpositions via Nested Interferometry, Phys. Rev. Lett. 109, 023601 (2012).

[25] D. E. Chang, C. A. Regal, S. B. Papp, D. J. Wilson, J. Ye, O. Painter, H. J. Kimble, and P. Zoller, Cavity opto-mechanics using an optically levitated nanosphere, Proc. Natl. Acad. Sci. U.S.A. 107, 1005 (2010).

[26] O. Romero-Isart, A. C. Pflanzer, F. Blaser, R. Kaltenbaek, N. Kiesel, M. Aspelmeyer, and J. I. Cirac, Large Quantum Superpositions and Interference of Massive NanometerSized Objects, Phys. Rev. Lett. 107, 020405 (2011).

[27] A. Albrecht, A. Retzker, and M. B. Plenio, Testing quantum gravity by nanodiamond interferometry with nitrogen-vacancy centers, Phys. Rev. A 90, 033834 (2014).

[28] N. Bar-Gill, L. M. Pham, A. Jarmola, D. Budker, and R. L. Walsworth, Solid-state electronic spin coherence time approaching one second, Nat. Commun. 4, 1743 (2013).

[29] M. H. Abobeih, J. Cramer, M. A. Bakker, N. Kalb, M. Markham, D. J. Twitchen, and T. H. Taminiau, One-second coherence for a single electron spin coupled to a multi-qubit nuclear-spin environment, Nat. Commun. 9, 2552 (2018).

[30] M. W. Doherty, N. B. Manson, P. Delaney, F. Jelezko, J. Wrachtrup, and L. C. L. Hollenberg, The nitrogen-vacancy colour centre in diamond, Phys. Rep. 528, 1 (2013).

[31] Y. Wu, F. Jelezko, M. B. Plenio, and T. Weil, Diamond quantum devices in biology, Angew. Chem., Int. Ed. Engl. 55, 6586 (2016).

[32] L. P. Neukirch, J. Gieseler, R. Quidant, L. Novotny, and A. N. Vamivakas, Observation of nitrogen vacancy photoluminescence from an optically levitated nanodiamond, Opt. Lett. 38, 2976 (2013).

[33] L. P. Neukirch, E. von Haartman, J. M. Rosenholm, and A. N. Vamivakas, Multi-dimensional single-spin nano-optomechanics with a levitated nanodiamond, Nat. Photonics $\mathbf{9}$, 653 (2015).

[34] R. M. Pettit, L. P. Neukirch, Y. Zhang, and A. N. Vamivakas, Coherent control of a single nitrogen-vacancy center spin in optically levitated nanodiamond, J. Opt. Soc. Am. B 34, C31 (2017).

[35] T. M. Hoang, J. Ahn, J. Bang, and T. Li, Electron spin control of optically levitated nanodiamonds in vacuum, Nat. Commun. 7, 12250 (2016).

[36] A. Kuhlicke, A. W. Schell, J. Zoll, and O. Benson, Nitrogen vacancy center fluorescence from a submicron diamond cluster levitated in a linear quadrupole ion trap, Appl. Phys. Lett. 105, 073101 (2014).

[37] T. Delord, L. Nicolas, L. Schwab, and G. Hétet, Electron spin resonance from $\mathrm{NV}$ centers in diamonds levitating in an ion trap, New J. Phys. 19, 033031 (2017).

[38] T. Delord, P. Huillery, L. Schwab, L. Nicolas, L. Lecordier, and G. Hétet, Ramsey Interferences and Spin Echoes from Electron Spins inside a Levitating Macroscopic Particle, Phys. Rev. Lett. 121, 053602 (2018).

[39] G. P. Conangla, A. W. Schell, R. A. Rica, and R. Quidant, Motion control and optical interrogation of a levitating single nitrogen vacancy in vacuum, Nano Lett. 18, 3956 (2018).

[40] T. Delord, P. Huillery, L. Nicolas, and G. Hétet, Spincooling of the motion of a trapped diamond, Nature (London) 580, 56 (2020).

[41] J.-F. Hsu, P. Ji, C. W. Lewandowski, and B. D’Urso, Cooling the motion of diamond nanocrystals in a magneto-gravitational trap in high vacuum, Sci. Rep. 6, 30125 (2016).

[42] M. C. O'Brien, S. Dunn, J.E. Downes, and J. Twamley, Magneto-mechanical trapping of micro-diamonds at low pressures, Appl. Phys. Lett. 114, 053103 (2019).

[43] C. Wan, M. Scala, G. W. Morley, A. T. M. A. Rahman, H. Ulbricht, J. Bateman, P. F. Barker, S. Bose, and M. S. Kim, Free Nano-Object Ramsey Interferometry for Large Quantum Superpositions, Phys. Rev. Lett. 117, 143003 (2016).

[44] S. Bose and G. W. Morley, Matter and spin superposition in vacuum experiment (MASSIVE), arXiv:1810.07045.

[45] M. Scala, M. S. Kim, G. W. Morley, P. F. Barker, and S. Bose, Matter-Wave Interferometry of a Levitated Thermal Nano-Oscillator Induced and Probed by a Spin, Phys. Rev. Lett. 111, 180403 (2013).

[46] Z.-q. Yin, T. Li, X. Zhang, and L. M. Duan, Large quantum superpositions of a levitated nanodiamond through spinoptomechanical coupling, Phys. Rev. A 88, 033614 (2013).

[47] J. S. Pedernales, G. W. Morley, and M. B. Plenio, Noise analysis in diamond based matter-wave interferometers (to be published). 